ACUTE CORONARY SYNDROMES

\title{
Risk of acute MI with NSAID use
}

Oral nonsteroidal anti-inflammatory drug (NSAID) use is associated with an increased risk of acute myocardial infarction (MI) according to a meta-analysis published in the BMJ. "Risk was greatest during the first month of NSAID use and with higher doses," report the researchers.

The Bayesian meta-analysis of individual patient data included 446,763 individuals from Canada and Europe, 61,460 of whom had an MI. The drugs studied were the traditional NSAIDs diclofenac, ibuprofen, and naproxen, and the cyclooxygenase 2-selective NSAIDs celecoxib and rofecoxib.

An increased risk of $\mathrm{Ml}$ was found with short-term (1-7 day) use of rofecoxib (OR 1.58, $95 \% \mathrm{Cl} 1.07-2.17$ ), naproxen (OR $1.53,95 \% \mathrm{Cl}$ 1.07-2.33), diclofenac (OR $1.50,95 \% \mathrm{Cl}$ 1.06-2.04), ibuprofen (OR 1.48, 95\% Cl 1.00-2.26), and celecoxib (OR 1.24, 95\% Cl 0.91-1.82). Of note, the risk of $\mathrm{Ml}$ increased immediately with exposure to NSAIDs.

Higher doses of NSAIDs (celecoxib $>200 \mathrm{mg}$, diclofenac $>100 \mathrm{mg}$, ibuprofen $>1,200 \mathrm{mg}$, naproxen $>750 \mathrm{mg}$, and rofecoxib $>25 \mathrm{mg}$ ) were associated with greater risks of $\mathrm{MI}$. With prolonged use of NSAIDs, the risk of $\mathrm{MI}$ remained constantly elevated, but did not seem to increase further; however, data on the risk of a second MI were not available. In summary, all NSAIDs - including naproxen (which had previously been thought to have superior cardiovascular safety) are associated with an increased risk of MI. "Given that the onset of risk of acute MI occurred in the first week and appeared greatest in the first month of treatment with higher doses," comment the researchers, "prescribers should consider weighing the risks and benefits of NSAIDs before instituting treatment, particularly for higher doses."

Gregory B. Lim

ORIGINAL ARTICLE Bally, M. et al. Risk of acute myocardial infarction with NSAIDs in real world use: bayesian meta-analysis of individual patient data. BMJ 357, j1909 (2017)

FURTHER READING González-Gay, M. A. \& GonzálezJuanatey, C. Inflammtion: NSAIDs and cardiovascular risk in arthritis. Nat. Rev. Cardiol. 14, 69-70 (2017)

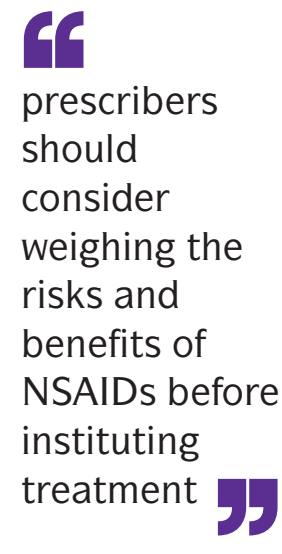

prescribers

should

consider

weighing the

risks and

benefits of

NSAIDs before

instituting 